\title{
Threshold Levels of Sperm Parameters Impacting on Pregnancy Rate in an Intrauterine Insemination Programme
}

\author{
Moez Kdous ${ }^{1, ~ ", ~ F e t h i ~ z h i o u a ~}{ }^{1}$, Amel Zhioua ${ }^{2}$ \\ ${ }^{1}$ Department of Gynecology and Obstetrics, Aziza Othmana Hospital, Medicine School of Tunis, University of Tunis El Manar, Tunis, \\ Tunisia \\ ${ }^{2}$ Department of Reproductive Biology, Aziza Othmana Hospital, Medicine School of Tunis, University of Tunis El Manar, Tunis Tunisia
}

Email address:

kdousmoez@gmail.com (M. Kdous)

\section{To cite this article:}

Moez Kdous, Fethi zhioua, Amel Zhioua. Threshold Levels of Sperm Parameters Impacting on Pregnancy Rate in an Intrauterine Insemination Programme. Journal of Gynecology and Obstetrics. Vol. 3, No. 5, 2015, pp. 98-102. doi: 10.11648/j.jgo.20150305.11

\begin{abstract}
Objectives. Intrauterine insemination (IUI) is frequently used as a first line strategy in the treatment of male and unexplained infertility. Threshold levels of sperm parameters associated with IUI success are controversial. The aim of this study was to evaluate the influence of sperm parameters on the outcome of IUI. Study Design. A prospective observational study. Patients and methods. This study included 295 IUI cycles. All IUI cycles were preceded by ovarian superovulation with clomiphene citrate $50 \mathrm{mg}$ tablets orally twice daily for 5 days starting on the second day of menses and recombinant FSH 150 units IM on the 6th, 8th, and 10th day. Cycles were monitored by transvaginal ultrasound. The IUI was performed with a catheter $36 \pm 4$ hours after hCG injection. Sperm parameters before and after semen treatment for IUI were evaluated and correlated with pregnancy outcome. Results. A total of 29 clinical pregnancies were obtained, for a pregnancy rate per cycle of $9.83 \%$. No pregnancy was obtained when less than one million spermatozoa were inseminated $(p=0.022)$. A statically significant increase in pregnancy rate was observed when normal sperm morphology was $>20 \%$ before semen treatment $(p=$ $0.01)$ and $>25 \%$ after semen treatment $(\mathrm{p}=0.034)$. NTSI (number of typical spermatozoa inseminated) and NTMSI (number of typical and mobile spermatozoa inseminated) significantly influence the clinical pregnancy rate. A $65 \%$ decrease in pregnancy rate was observed when the NTSI $<2106$ compared to the NTSI $\geq 2106(5.16 \%$ vs $15 \%, p=0.004)$. Similarly, a 54\% decrease in pregnancy rate was observed when the NTMS $<106$ " compared to the NTMS $\geq 106(6.12 \%$ vs $13.5 \%, p=0.026)$. Conclusion. IUI used for treating male factor infertility seems to have little chance of success when NMSI $<1 \times 106$, NTSI $<2$ million, NTMSI $<1$ million. If theses thresholds cannot be obtained, IVF should be recommended.
\end{abstract}

Keywords: Intrauterine Insemination, Sperm Parameters, Morphology, Motility, Pregnancy Rate

\section{Introduction}

Intrauterine insemination (IUI) is the simplest, least invasive and least expensive of the Assistance Reproductive Technologies (ART). Cervical infertility, male and unexplained infertility are still the major indications of this procedure [1].However, the success rates of this technique are still low, suggesting that IUI should be retained only when it is medically indicated and biologically feasible.

There are certain variables that are currently known to be predictive of IUI success, most of which relate to the female partner, no male factors were found to correlate with the treatment outcome. There is only one accepted male semen analysis parameter, either from the pre or post processing analysis, which has been shown to be predictive of IUI outcome. That parameter total motile sperm count [2]. Data from the literature are contradictory and do not allow to identify clearly if other sperm parameters before or after semen treatment are associated with IUI success and which minimum threshold values should be accepted to use in daily practice in order to be able to establish a real guide of good practices for insemination [3].

The evaluation of our practices was therefore necessary so as to offerable patients an adapted and personalized management. The aim of our study was to determine the impact of the sperm parameters on the success of IUI.

\section{Patients and Methods}

From January 2006 to December 2009, 295 couples (women younger than 40 years of age), all at their first 
attempt, completed 295 IUI cycles for male factor infertility in our department of biology and reproductive medicine. Institutional Review Board approval was obtained for this study. Before resorting to IUI, all women were subjected to testing for tubal patency by hysterosalpingography, and evaluating the pelvic anatomy by transvaginal ultrasonography. Laparoscopy was done when there was a possibility of pelvic adhesions or endometriosis in the HSG or transvaginal ultrasonography. The assessment of the ovarian reserve was performed in all patients by a hormonal dosage done on day 3 of the cycle (FSH, LH, E2) associated to an ultrasound antral follicles count. Men had at least two semen analyses and microbiological tests before any treatment. All couples were tested for hepatitis B virus and hepatitis $C$ virus before they were offered the IUI trial. Additional testing depended on any abnormalities observed.

Normal semen analyses were defined by the threshold values of the World Health Organization [4](concentration $20 \times 106 / \mathrm{mL}$, total count $40 \times 106$, progressive motility $50 \%$, and typical morphology $30 \%$ ). IUI was indicated only if at least $10^{6}$ progressive motile spermatozoa were selected during a migration survival test. All semen samples were collected in the laboratory after 3 to 5 days of sexual abstinence. After liquefaction for 30 minutes at room temperature, volume, $\mathrm{pH}$, sperm count, and progressive motility " $a+b$ " were evaluated according to the WHO standard criteria. Sperm concentration was performed with a hemocytometer on two separate preparations of the semen sample (dilution 1:20 in Ringer's solution). Sperm motility was determined by assessing at least five microscopic fields to classify at least 200 spermatozoa ( $\times 4,000$ magnifications). The motility was graded progressive, nonprogressive, or immotile. Motile sperm were selected by a swim-up procedure. In all cases, the motile sperm fraction was washed twice by centrifugation, and the sperm pellet was suspended in $0.35 \mathrm{~mL}$ of Earle's balanced salt solution (Sigma-Aldrich Co., Ayrshire, United Kingdom) as a capacitating medium in all patients. Sperm were then counted, and progressive motility assessed.To analyze sperm morphology, smears were prepared from the whole ejaculated fraction and from the motile selected one. The staining procedure was carried out according to the Spermac kit manufacturer's guidelines (Stain Enterprises, Onderstepoort, South Africa). The sperm smear was allowed to air-dry before being fixed in the formalin solution (fixative I) provided in the Spermac kit for 5 minutes at room temperature $\left(23^{\circ} \mathrm{C}\right)$. Each slide was flooded with stain solution A for 2 minutes and then rinsed off with water. Each slide was then flooded with stain solution $\mathrm{B}$ and $\mathrm{C}$ each for 1 minute successively. The percentages of morphologically normal spermatozoa and of various sperm abnormalities were evaluated on 100 sperm at $\times 1,000$ magnification according to criteria of David and al. [5] modified by Jouannet and al. [6]

Ovarian stimulation was performed in all the patients with clomiphene citrate (Clomid $\AA$, Sanofi-Aventis, France) $100 \mathrm{mg} / \mathrm{d}$ from 2nd to 6th day of the cycle and recombinant FSH (GonalF $®$, Serono, France) 75 or 150 units on the 6 th, 8 th, and 10 th day. Cycles were monitored by transvaginal ultrasound for the mean follicular volume and thickness of the endometrium on days 10, 12, and 14 of the cycle. Human chorionic gonadotropin 5,000 IU injection was given to induce ovulation when at least one follicle measured $18 \mathrm{~mm}$ or more. The IUI was performed with a catheter (Frydmancatheter $\left.{ }^{(}\right)(C C D$, France) $36 \pm 5$ hours after hCG injection. All patients received $400 \mathrm{mg} /$ day of dydrogesterone (Utrogestan $\AA$, International Besins, France) after insemination till the day of hCG testing. Serum hCG was determined 2 weeks after hCG injection for diagnosis of pregnancy. The outcome measure was the occurrence of clinical pregnancy, diagnosed by the visualization of fetal cardiac activity on ultrasound scan.

Data are expressed as the mean \pm SD or percentages. The $\chi 2$-test, Fisher's exact test, and Student's $t$-test were used for statistical analysis with SPSS software, version 15.0 for Windows (SPSS, Inc, Chicago, IL, USA). $P<.05$ was considered statistically significant.

\section{Results}

The mean age of the women at IUI was $33.82 \pm 5.074$ years (range 23-40 years). According to the WHO criteria [4], various sperm abnormalities were encountered such as isolated oligospermia in 18 patients $(6 \%)$, isolated asthenospermia in 12 patients $(4 \%)$, isolated teratospermia in 65 patients $(22 \%)$, oligoasthenospermia in 9 patients $(3 \%)$, oligoteratospermia in 6 patients $(2 \%)$, asthenoteratospermia in 36 patients $(12 \%)$, and oligoasthenoteratospermia in 40 patients $(13 \%)$.

Twenty-nine clinical pregnancies followed 295 IUI cycles, for a clinical pregnancy rate of $9.83 \%$. Spontaneous early abortions occurred in 11 pregnancies (3.7\%), and twin pregnancies occurred in 4 patients $(1.3 \%)$.

Table 1. Sperm parameters values observed in both pregnant and non-pregnant groups before and after semen treatment.

\begin{tabular}{lllllll}
\hline & Before semen treatment & & \multicolumn{3}{c}{ After semen treatment } \\
\hline & Non pregnant $(\mathbf{n = 2 6 6})$ & Pregnant $(\mathbf{n}=\mathbf{2 9})$ & $\mathbf{P}$ & Non pregnant $(\mathbf{n = 2 6 6})$ & Pregnant $(\mathbf{n = 2 9 )}$ & P \\
\hline Concentration (106/ml) & $68.16 \pm 18.1$ & $53.45 \pm 17.8$ & .288 & $24.26 \pm 10.5$ & $25.59 \pm 11.2$ & .732 \\
Motility « a » (\%) & $13.44 \pm 6.3$ & $16.21 \pm 7.2$ & .132 & $36.02 \pm 8.3$ & $41.38 \pm 9.1$ & .111 \\
Motility « b » (\%) & $29.52 \pm 15.4$ & $30.52 \pm 14.6$ & .592 & $35.17 \pm 10.3$ & $34.66 \pm 10.5$ & .806 \\
Motility « a+b » (\%) & $42.96 \pm 20.6$ & $46.72 \pm 19.8$ & .178 & $73.12 \pm 18.6$ & $76.03 \pm 19.1$ & .121 \\
Typical form (\%) & $17.98 \pm 7.4$ & $24.93 \pm 8.2$ & .001 & $22.15 \pm 8.6$ & $29.76 \pm 7.7$ & .005 \\
\hline
\end{tabular}


Table 2. Pregnancy rate correlated to sperm parameters values before and after semen treatment.

\begin{tabular}{|c|c|c|c|c|c|c|c|c|}
\hline & \multicolumn{4}{|c|}{ Before semen treatment } & \multicolumn{4}{|c|}{ After semen treatment } \\
\hline & $\%$ & cycles & Pregnancy n (\%) & $\mathbf{P}$ & $\%$ & cycles & Pregnancy n (\%) & $\mathbf{P}$ \\
\hline \multirow[t]{2}{*}{ Motility «a » $(\%)$} & $<15$ & 149 & $10(6.7)$ & \multirow[t]{2}{*}{.052} & $<35$ & 128 & $9(7.03)$ & \multirow{2}{*}{.111} \\
\hline & $\geq 15$ & 146 & $19(13.01)$ & & $\geq 35$ & 167 & $20(11.97)$ & \\
\hline \multirow[t]{2}{*}{ Motility « $\mathrm{a}+\mathrm{b} »(\%)$} & $<50$ & 170 & $15(8.82)$ & \multirow[t]{2}{*}{.314} & $<75$ & 129 & $12(9.3)$ & \multirow[t]{2}{*}{.474} \\
\hline & $\geq 50$ & 125 & $14(11.2)$ & & $\geq 75$ & 166 & $17(10.2)$ & \\
\hline Typical form (\%) & $\begin{array}{l}<20 \\
\geq 20\end{array}$ & $\begin{array}{l}167 \\
128\end{array}$ & $\begin{array}{l}10(5.9) \\
19(14.84)\end{array}$ & .010 & $\begin{array}{l}<25 \\
\geq 25\end{array}$ & $\begin{array}{l}154 \\
141\end{array}$ & $\begin{array}{l}10(6.4) \\
19(13.4)\end{array}$ & .034 \\
\hline
\end{tabular}

Table 3. Comparison of NMSI, NTSI and NTMSI between pregnant and nonpregnant groups.

\begin{tabular}{llll}
\hline & Non pregnant $(\mathbf{n}=\mathbf{2 6 6})$ & Pregnant $(\mathbf{n}=\mathbf{2 9})$ & $\mathbf{P}$ \\
\hline NMSI (106) & 5.3 & 5.45 & .877 \\
NTSI (106) & 3.42 & 3.45 & .977 \\
NTMSI (106) & 1.65 & 1.34 & .471 \\
\hline
\end{tabular}

NMSI: number of motile spermatozoa inseminated; NTSI: number of typical spermatozoa inseminated;

NTMSI: number of typical and motile spermatozoa inseminated.

Table 4. Pregnancy rate correlated to NMSI, NTSI, NMTSI.

\begin{tabular}{lllll}
\hline & & cycles & Pregnancy n (\%) & P \\
\hline NMSI (106) & $<1$ & 35 & $0(0)$ & .022 \\
& $1 \leq \mathrm{N}<5$ & 119 & $14(11.76)$ & .980 \\
& $5 \leq \mathrm{N}<10$ & 99 & $11(11.11)$ & \\
& $\mathrm{N} \geq 10$ & 42 & $4(9.52)$ & \\
NTSI (106) & $<2$ & 155 & $8(5.16)$ & $.004 \mathrm{a}$ \\
& $\geq 2$ & 140 & $21(15)$ & $.026 \mathrm{~b}$ \\
NTMSI (106) & $<1$ & 147 & $10(6.12)$ & \\
& $\geq 1$ & 148 & $19(13.5)$ & \\
\hline
\end{tabular}

NMSI: number of motile spermatozoa inseminated, NTSI: number of typical spermatozoa inseminated; NTMSI: number of typical and motile spermatozoa inseminated; a a $65 \%$ decrease in pregnancy rate; b a $54 \%$ decrease in pregnancy rate.

Table 1 shows no significant difference in: total sperm concentration, motility 'a', 'b' and "a + b" before and after sperm treatment, between patients who achieved pregnancy and those who failed. Only normal sperm morphology was significantly associated to pregnancy rate $(\mathrm{p}=0.001$ before sperm treatment and 0.005 after)

Table 2 shows a statically significant increase in pregnancy rate when normal sperm morphology was $>20 \%$ before semen treatment $(p=0.01)$ and $>25 \%$ after semen treatment $(\mathrm{p}=0.034)$.

Comparison of NMSI (number of motile spermatozoa inseminated), NTSI (number of typical spermatozoa inseminated) and NTMSI (number of typical and motile spermatozoa inseminated) between pregnant and nonpregnant groups showed no significant differences (table 3 ).

No pregnancy was obtained when less than one million spermatozoa were inseminated $(p=0.022)$. Above one million, there is no statistical difference in pregnancy rates between subgroups $1 \leq \mathrm{NMSI}<5 ; 5 \leq \mathrm{NMSI}<10$ and NMSI $\geq 10$. Beyond 10 million, our results showed a slight decline (though not significant).

NTSI and NTMS significantly influence the occurrence of clinical pregnancy. A $65 \%$ decrease in pregnancy rate was observed when the NTSI $<210^{6}$ compared to the NTSI $\geq 2$
$10^{6}(5.16 \%$ vs $15 \%, p=0.004)$. Similarly, a $54 \%$ decrease in pregnancy rate was observed when the NTMS $<10^{6}$, compared to the NTMS $\geq 10^{6}(6.12 \%$ vs $13.5 \%, p=0.026)$. (Table 4)

\section{Discussion}

There is good evidence in literature that intrauterine insemination (IUI) is the best first line treatment and most cost-effective procedure for moderate male factor subfertility. It seems very difficult to identify individual semen parameters predicting the likelihood of pregnancy after IUI. This can be explained by a lack of standardization of semen analysis, but many other methodological variables may also influence IUI success rates such as the patient selection, type of ovarian stimulation and number of inseminations per cycle [7].

The majority of studies attempting to find which sperm characteristics correlated better with cycle outcome included multiple female infertility problems as well. Hence, the results might be biased by the other infertility etiologies that were treated in parallel. In this study, only the male factor problems were recruited to make the differences in sperm parameters more significant. Twenty-nine clinical pregnancies were achieved after 295 IUIs (9.83\%). In a review of 17 papers about IUI with ovarian stimulation by hMG, Wainer et al. [8] reported 274 pregnancies in 2,223 IUI cycles, for a pregnancy rate per cycle of $12.33 \%$.

It has previously been demonstrated that, the number of motile spermatozoa inseminated (NMSI) and sperm morphology are the most valuable sperm parameters to Predict IUI outcome.The minimum recommended number of motile sperm after semen treatment in various reports varies from 0.8 to $10 \times 10^{6}[9,10,11$ and 12]. In the study of Badaway and al [13] the pregnancy rate per cycle was significantly lower in the subgroups with number of motile sperm $<5 \times 106(5.55 \%$ vs. $24.8 \%)$. This was in agreement with Francavilla and al. [14], who found a significant increase in success rate when the total motile sperm count exceeded $5 \times 10^{6}$. Van Weert and al [15] in a meta-analysis of 16 studies reported a cut-off of NSMI between 0.8 and 5 million. In this study, the number of motile sperm inseminated (NMSI) was not significantly different in the cycles resulting in pregnancy compared to the failed cycles. But, when (NMSI) was $<1 \times 10^{6}$, no pregnancy was obtained in our study $(\mathrm{p}<0.05)$. However, the low number of attempts with less than 1 million NMSI generated a sampling bias limiting the relevance of this parameter. Beyond 1 million, 
the NSMI does not seems to influence pregnancy rate significantly. But, when NSMI exceeded 10 million, our results showed a slight non-significant decrease in pregnancy rate, which could be related to excessive production of free radicals by the spermatozoa. Most authors recommend IVF when the number of motile sperm is $<1 \times 10^{6}[11,12,16$ and 17]Some authors suggested using the total number of motile spermatozoa of the initial sperm count (before semen treatment) as the criterion for choosing between IUI and IVF, and have recommended threshold values ranging from 5 to $10 \times 10^{6}[18,19]$. In other studies, total sperm motility before semen treatment was mentionedwith a cut-off level between 30 and $50 \%$ [20,21, 22 and 23].The usefulness of this criterion, however, is limited by the variability of quality from one ejaculation to another, as well as the variable results of sperm preparations.Our study shows no significant difference in sperm motility before sperm treatment, between patients who achieved pregnancy and those who failed

Sperm morphology using strict criteria is well known as one of the best predictors of IVF outcome[24, 25 and 26]. Sperm morphology has also turned out to be a good predictive parameter in IUI. In a meta-analysis, a significant improvement in pregnancy rates above the $4 \%$ threshold for strict criteria was described [15]. Universally, the most common accepted classification system used for sperm morphology is the WHO criteria [20]. The threshold of spermatozoa with normal morphology below which IVF is recommended varies widely in the literature from $4 \%$ to $50 \%$ [27, 28, 29 and 30]. The present analysis supported these results (thresholds of $20 \%$ in the initial sperm and $25 \%$ after semen treatment)

However, the implication of sperm morphology alone for choice and outcome of IUI is controversial. Many retrospective and prospective studies showed that sperm morphology alone, before or after preparation, did not help to predict IUI results [31, 32 and 33]. The evaluation of number of motile sperm in conjunction with sperm morphology might represent an important deliberation for clinical decision making. According to Wainer and al [8] For a normal morphology sperm rate $<30 \%$, the clinical pregnancy rate was $4.62 \%$ when the number of motile sperm inseminated (NMSI) was $<5 \times 10^{6}$ and $9.45 \%$ when the NMSI was $>5 \times 10^{6}$, and this difference was statistically significant, suggesting a partial compensation of quality by the amount of spermatozoa. The study of Badawy and al [13] showed that above 5 million motile spermatozoa inseminated, the clinical pregnancy rate per cycle was $5.3 \%$ when the normal morphology sperm rate (NTSI) rate $<30 \%$ and $18.42 \%$ when $>30 \%$.Our study supported this findings and showed that the NTSI and the NTMSI significantly influence the pregnancy rate in IUI. Thus, when the NTSI was less than 2 million, IUI proved to be an inefficient ART indication with a pregnancy rate of only $5.16 \%$. In contrast, when the NTSI was greater than or equal to 2 million pregnancy rate increases to $15 \%(\mathrm{p}=0.04)$.

Similarly, when the NTMSI was less than 1 million, the success rate of IUI was $6.12 \%$. This rate increased to $13.5 \%$ when the NTMSI was greater than or equal to 1 million $(\mathrm{p}=$ 0.026).

\section{Conclusion}

In view of these results, IUI used for treating male factor infertility seems to have little chance of success when NMSI $<1 \times 10^{6}$, NTSI $<2$ million, NTMSI $<1$ million. If theses thresholds cannot be obtained, IVF should be recommended. In this study 295 inseminations cycles were evaluated, it is possible that the sample size may not be enough to detect statistical significance for some parameters with small differences expected in the groups. Further prospective trials will help elucidate the literature. Furthermore, these trials should control for confounding parameters

\section{References}

[1] Marchetti C and Dewailly D. Intrauterine insemination: indications and methods. Rev Prat 2006; 56(5): 500-6.

[2] Luco SM, Agbo C, Behr B, Dahan MH. The evaluation of pre and post processing semen analysis parameters at the time of intrauterine insemination in couples diagnosed with male factor infertility and pregnancy rates based on stimulation agent. A retrospective cohort study. Eur J Obstet Gynecol Reprod Biol 2014 (179): 159-162.

[3] Royère D. Insémination intra-utérine: état des lieux chez l'humain. Gynécol Obstet Fertil 2004; 32(10) 873-9.

[4] World Health Organization. Laboratory manual for the examination and processing of human semen. Fourth edition. 1999.

[5] David G, Bisson JP, Czyglik F, Jouannet P, Gernigon C. Anomalies morphologiques du spermatozoïde humain. Proposition pour un système de classification. J Gynecol Obstet Biol Reprod 1975; 4 : 17-36.

[6] Jouannet P, Ducot B, Feneux D, Spira A. Male factors and the likelihood of pregnancy in infertile couples. Study and sperm characteristics. Int J Androl 1988; 11: 379-384.

[7] Ombelet W, Deblaere K, Bosmans E, and al. Semen quality and intrauterine insemination. Reprod Bio Med Online 2003 $4: 485-492$.

[8] Wainer $R$, Albert $M$, Dorion A, Bailly $M$, Bergère $M$, Lombroso $\mathrm{R}$, and al. Influence of the number of motile spermatozoa inseminated and of their morphology on the success of intrauterine insemination. Hum Reprod 2004; 19: 2060-2065.

[9] Dodson WC, Haney AF. Controlled ovarian hyperstimulation and intrauterine insemination for treatment of infertility. Fertil Steril 1991; 55: 457-467.

[10] Berg U, Brucker C, Berg FD. Effect of motile sperm count after swim-up on outcome of intrauterine insemination. Fertil Steril 1997; 67: 747-750.

[11] Van Voorhis BJ, Barnett M, Sparks AET, Syrop CH, Rosenthal G, Dawson J. Effect of the total motile sperm count on the efficacy and cost-effectiveness of intrauterine insemination and in vitro fertilization. Fertil Steril 2001; 75: 661-668. 
[12] Miller DC, Hollenbeck BK, Smith GD, Randolph JF, Christman GM, Smith YR, and al. Processed total motile sperm count correlates with pregnancy outcome after intrauterine insemination. Urology 2002: 497-501.

[13] Badawy A, Elnashar A, Eltotongy M. Effect of sperm morphology and number on success of intrauterine insemination. Fertil Steril 2009; 91:777-781.

[14] Francavilla F, Romano R, Santucci R, Poccia G. Effect of sperm morphology and motile sperm count on outcome of intrauterine insemination in oligozoospermia and/or asthenospermia. Fertil Steril 1990, 53: 892-897.

[15] Van Waart J, Kruger TF, Lombard CJ, Ombelet W. Predictive value of normal sperm morphology in intra uterine insemination (IUI): a structured literature review. Hum Reprod Update 2001; 7(5): 495-500.

[16] Brasch JG, Rawlins R, Tarchala S, Radwanska E. The relations between total motile sperm count and the success of intrauterine insemination. Fertil Steril 1994; 62: 150-154.

[17] Horvath P, Bohrer M, Shelden R, Kemmann E. The relationship of sperm parameters to cycle fecundity in superovulated woman undergoing intrauterine insemination. Fertil Steril 1989, 52: 288-294.

[18] Campana A, Sakkas D, Stalberg A, Grace Bianchi P, Comte I, Pache $\mathrm{T}$, and al. Intrauterine insemination: evaluation of the results according to the woman's age, sperm quality, total sperm count per insemination and life table analysis. Hum Reprod 1996; 11: 732-736.

[19] Elvan Koyun Ok, Ömer Erbil Doğan, Recep Emre Okyay, and Bülent Gülekli. The effect of post-wash total progressive motile sperm count and semen volume on pregnancy outcomes in intrauterine insemination cycles: a retrospective study. J Turk Ger Gynecol Assoc2013; 14(3): 142-145.

[20] Shulman A, Hauser R, Lipitz S and al. Sperm motility is a major determinant of pregnancy outcome following intrauterine insemination Journal of Assisted Reproduction and Genetics 1998; 15: 381-385.

[21] Dickey RP, Pyrzak R, Lu PY and al. Comparison of the sperm quality necessary for successful intrauterine insemination with World Health Organization threshold values for normal sperm. Fertil Steril 1999; 71, 684-689.

[22] Montanaro GM, Kruger TF, Coetzee K and al. Stepwise regression analysis to study male and female factors impacting on pregnancy rate in an intrauterine insemination programme. Andrologia 2001; 33 : 135-141.
[23] Lee RK-K, Hou J-W, Ho H-Y and al. Sperm morphology analysis using strict criteria as a prognostic factor in intrauterine insemination. International Journal of Andrology 2002; 25: 277-280.

[24] Cao S, Zhao C, Zhang J, Wu X, Zhou L, Guo X and al. A minimum number of motile spermatozoa are required for successful fertilisation through artificial intrauterine insemination with husband's spermatozoa. Andrologia 2014; 46 (5): 529-534.

[25] Saucedo de la Llata E, Moraga Sanchez M, Batiza Resendiz V, Galache Vega P, Santos Haliscak R, Hernandez Ayup S. Influence of sperm morphology on results of intrauterine insemination. Ginecol Obstet Mex 2003; 71: 455-9.

[26] Sun Y, Li B, Fan LQ et al. Does sperm morphology affect the outcome of intrauterine insemination in patients with normal sperm concentration and motility? Andrologia 2012; 44(5): 299-304.

[27] Kruger TF, Menkveld R, Stander FSH, Lombard CJ, van Zyl J, Smith K. Sperm morphologic features as a prognostic factor in in vitro fertilization. Fertil Steril 1986; 46: 1118-1123.

[28] Sakhel K, Abozaid T, Schwarck S, Ashraf M, and al Semen parameters as determinants of success in 1662 cycles of intrauterine insemination Effective controlled ovarian hyperstimulation Fertil Steril 2005; 84:248-249.

[29] Haim D, Leniaud L, Porcher R, Martin-Pont B, Wolf JP, Sifer C. Prospective evaluation of the impact of sperm characteristics on the outcome of intrauterine inseminations. Gynecol Obstet Fertil 2009; 37:229-235.

[30] Berker B, Sükür YE, Kahraman K Atabekoglu CS, Sonmezer M, Ozmen and al Absence of Rapid Linear and Progressive Motile Spermatozoa "Grade A" in Semen Specimens: Does It Change Intrauterine Insemination Outcomes? Urology 2012; 80(6): 1262-6.

[31] Hassa H, Aydin Y, Oge T, Ozatik O, Taplamacioglu F. Predictive factors for clinical pregnancy in 354 unexplained infertile couple in their first intrauterine insemination cycle. Fertil Steril 2012; 98: 160.

[32] Matorras R, Corcostegui B, Perez C, Mandiola M, Mendoza R, Rodriguez-Escudero FJ. Sperm morphology analysis (strict criteria) in male infertility is not a prognostic factor in intrauterine insemination with husband's sperm. Fertil Steril 1995, 63: 608-611.

[33] Karabinus DS, Gelety DS. The impact of sperm morphology evaluated by strict criteria on intrauterine insemination success. Fertil Steril 1997; 67: 536-541. 